\title{
Inteligencia Práctica versus Inteligencia Artificial: El Futuro de la Acción Directiva en las Empresas
}

\section{Practical Reason vs. Artificial Intelligence: The Future of Business Management}

RECIBIDO: 8 DE OCTUBRE DE 2019/ACEPTADO: 18 DE NOVIEMBRE DE 2019

\section{RICARDO MURCIO RODRÍGUEZ}

Profesor Investigador, IPADE Business School (Mexico) rmurcio@ipade.mx

\section{JORGE LLAGUNO SAÑUDO}

Profesor Investigador, IPADE Business School (Mexico) jllaguno@ipade.mx

Resumen: Existe, en la actualidad, un debate en torno al futuro del trabajo, motivado en gran parte por la llamada Cuarta Revolución Industrial. En especial, por los desafíos que plantea la tecnología, y, de modo específico, la Inteligencia Artificial (IA). El desarrollo de la IA en los últimos años amenaza con reemplazar el trabajo humano, lo cual no solo acarrea consecuencias de orden social sino también antropológicas, por la importancia del trabajo en la vida de los hombres. El presente artículo compara la IA con la inteligencia práctica, o propiamente humana, con el fin de vislumbrar si tal amenaza es real. En primer lugar se realiza una aproximación teórica a la inteligencia práctica desde la filosofía clásica, tomando como referencia el marco teórico de Carlos Llano. Luego se contrasta con las percepciones sobre IA que surgen de entrevistas en profundidad realizadas a directivos de importantes empresas tecnológicas y profesionales del ámbito tecnológico en la ciudad de México.

Palabras clave: Inteligencia práctica, inteligencia artificial, acción directiva, trabajo, Carlos Llano.

\section{GERMÁN SCALZO \\ Profesor Investigador, Universidad Panamericana (Mexico) \\ german.scalzo@gmail.com}

\begin{abstract}
Debate abounds surrounding the future of work, motivated by the so-called Fourth Industrial Revolution, and especially by the challenges that technology and Artificial Intelligence (Al) pose. The development of Al in recent years threatens to replace human labor, which not only has social consequences, but also anthropological ones due to work's importance for human life. This paper compares Al with practical reason -i.e., properly human intelligence - in order to suss out if such a threat is real. First, it takes a theoretical approach to practical reason in light of classical philosophy, using Carlos Llano's theoretical framework as a reference. Then it contrasts this analysis with perceptions about Al gleaned from in-depth interviews conducted with executives and professionals at influential technology companies in Mexico City.
\end{abstract}

Keywords: Practical Reason, Artificial Intelligence, Management, Work, Carlos Llano. 


\section{INTRODUCCIÓN}

Existen en la actualidad importantes debates en torno al futuro del trabajo $^{1}$, promovidos por los complejos desafíos que plantea la llamada Cuarta Revolución Industrial, y en especial, la Inteligencia Artificial (IA), pero especialmente por una comprensión teórica muy limitada de la noción de trabajo humano ${ }^{2}$. Este debate es fundamental e ineludible, y no puede hacerse al margen de la sabiduría práctica, ni de la tecnología, pues, como destaca Vallor, "una teoría de que significa para los seres humanos la vida buena debe incluir una concepción explicita de cómo vivir bien con la tecnología"’3.

Dados los avances tecnológicos y los procesos deliberativos de las máquinas, es pertinente, y poco explorada, la pregunta acerca de si será posible prescindir de los servicios de los puestos directivos en las organizaciones, dejando esta labor en manos de ordenadores. El crecimiento en el poder de cómputo de los dispositivos móviles, aunado a la ubicuidad de las redes -ahora denominadas "la nube" - han abierto la puerta a su uso generalizado en todos los estratos, continentes y contextos. Es posible afirmar que los avances tecnológicos se encuentran hoy en día a la mano de todas las personas, y así como las computadoras en su momento cambiaron la manera de realizar las labores en las organizaciones, la posibilidad de delegar tareas no parametrizables implica ahora lo que se ha equiparado a una nueva revolución industrial, con sus previsibles consecuencias laborales y sociales. Estos cambios impactarán el trabajo de muchas personas en la empresa y es pertinente preguntarnos hasta dónde está previsto que la IA sea capaz de suplantar la labor directiva en las organizaciones del futuro.

Dentro del marco general del futuro del trabajo, esta investigación se pregunta sobre la posible sustitución del trabajo directivo en las organizaciones. Las comparaciones que se han realizado hasta el momento implican equivalencias entre el trabajo operativo y los avances tecnológicos ${ }^{4}$, aunque está empezando a ampliarse el ámbito de aplicación de la IA. Concretamente, queremos explorar si el tipo de decisiones que ejecuta una máquina puede sustituir la labor del director de empresa, utilizando como marco teórico la obra de Carlos Llano. Llano acude a la filosofía clásica para distinguir el trabajo directivo como aquella acción que no sigue reglas fijas y cuyo resultado es incierto, a diferencia del trabajo operativo que será aquel que sigue reglas fijas y

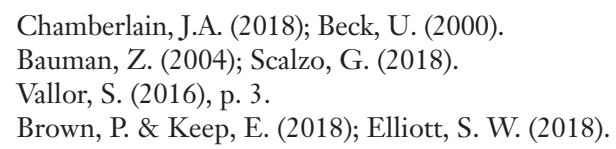


cuyos resultados son, siguiendo dichas reglas, al menos estadísticamente seguros 5 .

La labor directiva en las organizaciones se ha asociado a la 'inteligencia práctica' desde diferentes perspectivas ${ }^{6}$, siendo la prudencia (phronesis) su hábito más importante. El primero en distinguir los usos que se le pueden dar a la inteligencia fue Aristóteles, quien ya en la Ética a Nicómaco señala que la parte racional del ser humano puede dividirse en inteligencia teórica, cuya principal virtud es la sabiduría (sophia) e inteligencia práctica cuya principal virtud es la prudencia (phronesis). La clave para diferenciarlas radica en el tipo de realidades que captan: la inteligencia práctica se refiere a la posibilidad de captar la realidad en su sentido contingente, en contraposición de la inteligencia teórica que la aprecia de un modo abstracto, universal ${ }^{7}$.

Comenzaremos por delinear el alcance teórico de la inteligencia práctica en las organizaciones y posteriormente complementaremos esta reflexión con la percepción sobre la IA de profesionales del sector de la tecnología. Para ello hemos utilizado una metodología cualitativa, a saber, entrevistas en profundidad a seis directivos y expertos técnicos en el área de tecnología de cuatro empresas mexicanas líderes en el sector. Se trata de una primera aproximación exploratoria al tema, cuyo objetivo principal será comparar las capacidades de la IA entendiendo por ello, la situación actual de la tecnología respecto a la toma de decisiones en las empresas, sus límites y alcances, así como el futuro previsible, con las capacidades de la inteligencia práctica y su virtud prototípica la prudencia, específicamente en su aplicación a la dirección de empresas. Las investigaciones en este sentido plantean escenarios que van desde la reducción en algunas posiciones de trabajo hasta la desaparición de éste como lo entendemos hoy. En ningún caso se trata de futuros apocalípticos, en los que se cuestione el valor de la labor humana en la empresa, sino que en su mayoría abordan la necesidad de elevar las capacidades de los colaboradores para alcanzar niveles de toma de decisiones y, especialmente, de trabajo en equipo ${ }^{8}$.

5 Llano, C. (1990), p. 8.

6 Bachmann, C.; Habisch, A. \& Dierksmeie, C. (2017); Sison, A. \& Hühn, M. (2018).

7 Aristóteles, EN 1139a: "Ya hemos dicho que el alma tiene dos partes: la racional y la irracional. Pues bien, vamos asimismo a dividir esa parte racional, dejando establecido que, a su vez, son también dos sus partes: una la que utilizamos para contemplar los entes cuyos principios no pueden ser de otra manera y otra con la que contemplamos esa modalidad variable".

8 Brown, P. \& Keep, E. (2018). Elliott identifica que la IA no ha alcanzado los niveles 4 y 5 en la prueba Literacy PIA AC y, de acuerdo con su propia investigación, no prevé que lo logren en la próxima década. La aplicación de estos niveles a la empresa resulta, a nuestro entender, limitada si se confrontan con la Acción Directiva estudiada desde la Inteligencia Práctica. 


\section{LA INTELIGENCIA PRÁCTICA Y LA NATURALEZA DEL TRABAJO DIRECTIVO}

Antes de abordar si la dirección de empresas en las organizaciones está en riesgo, conviene definir la inteligencia práctica y su vínculo con la acción directiva. Para ello seguiremos mayormente a Carlos Llano, para quien la labor directiva en las empresas es una acción de síntesis entre asuntos aparentemente divergentes 9 , que no sigue reglas fijas y cuyos resultados son inciertos. Dadas estas peculiaridades, es preciso saber con qué facultad humana se pueden atender estos asuntos. La tradición aristotélico-tomista distingue entre razón teórica y razón práctica, como ya se referenció más arriba. Las dos diferencias más importantes entre estos tipos de pensamiento son: primero, que el pensamiento especulativo (estrictamente, el pensamiento científico de cualquier naturaleza) no trata sobre realidades en las que hay actuar, sino que pensar. Segundo, que este tipo de pensamiento consiste en una idealización de la realidad para estudiarla, y así, entenderla. El pensamiento para la acción -estrictamente, el pensamiento práctico-, trata sobre las realidades contingentes, para lo que debe atender las circunstancias concretas ${ }^{10}$.

Las distinciones fundamentales radican en dos aspectos: (1) la finalidad subjetiva de quién elabora la teoría, "la mente práctica difiere de la mente especulativa o teorética en el fin que persigue"; el fin de la teoría proviene de la persona que, por su voluntad, se lo señala, mientras que la especulativa es receptiva y capta el fin de la cosa misma que conoce; y, (2) la capacidad de llevar a la realidad lo pensado. La razón teórica no pretende modificar lo exterior, mientras que la práctica sí lo hace. La razón práctica necesita que la realidad

9 La esencia de la acción directiva radica en que "la función del director es una función de síntesis, de interrelación" (Llano, C. (1998), p. 21). "No ha de definirse, por tanto, la función del director por los dos elementos mencionados -decisión y mando- sino por el nivel desde el que esas dos acciones se ejecutan” (Llano, C. (1998), p. 44). Toma preponderancia, en la definición de acción directiva, la capacidad de interrelacionar diferentes aspectos de la realidad, ya se nota desde aquí una diferencia con la inteligencia artificial. Es posible considerar que el éxito en la gestión dependerá de la capacidad de sintetizar los aspectos aparentemente divergentes de la empresa, se desarrollan cinco aspectos (Llano, C. (1979), pp. 44-57): (1) Se capaz de dirigir las áreas funcionales a un mismo objetivo. (2) Ser capaz de unir el servicio a la comunidad con la ganancia económica. (3) Ser capaz de unir los objetivos de los distintos stakeholders. (4) Sintetizar el trabajo directivo y el operativo. (5) Lograr compaginar los aspectos de autoridad y poder en la dirección de personas.

10 Se trata de una distinción accidental, no son distintas potencias, sino una misma con diferentes fines. Aquino, STh, I ps., q. 79, a. 11, ad 1. Se añade otra referencia, razón teórica y razón práctica son una única potencia, puesto que las potencias del alma sólo se distinguen por razón de la diversa formalidad de sus objetos. Aquino, STh, I ps., q. 79, a. 7, co. 
sea trasformada, no pensada, por lo tanto, termina en la modificación de lo exterior por parte del sujeto; a diferencia del teórico o especulativo, que no modifica la realidad cuando aprehende, sino que pretende la adquisición de un conocimiento ${ }^{11}$. El director de empresa no pretende saber más, sino transformar la realidad, conseguir los resultados previstos. Hasta cierta medida se puede señalar que el cientificismo, racionalismo o mecanicismo de la empresa, no distingue estos dos tipos de razón, postulando que, desde la razón teórica, se puede modificar lo exterior. La labor del director no puede compararse con la de un científico, pretendiendo que el plan real sea igual al pensado. Esto sucede en las ciencias exactas, como las matemáticas, pero en la acción humana no pasa lo mismo. Desde el entendimiento teórico no se puede modificar la realidad, sólo conocerla, en abstracto, en lo que tiene de común con su especie.

Es importante entender esto, porque el director de empresa debe enfrentarse con problemas concretos que requieren soluciones del mismo carácter. Como resalta Llano, "[1]a empresa ha dejado de ser un mecanismo cuya dirección está ya previamente señalada, para convertirse, análogamente, en un organismo vivo, que requiere de un 'cerebro' rector en constante ejercicio, porque las variantes circunstancias externas le hacen cambiar constantemente de rumbo, y aun de objetivo"12. El director debe responder con acciones que transformen la realidad presente, y no con elucubraciones teóricas acerca de las causas de sus problemas. Con esto en mente, se comprende por qué el mero saber científico o estadístico es insuficiente, por ser estático ante una realidad cambiante, como lo es el mundo de la empresa. El director debe estar atendiendo las oportunidades que se presentan y no especulando sobre las razones por las cuales éstas se dan. La posibilidad de esta diferenciación entre el saber especulativo y el práctico es el punto de partida para entender la acción directiva como acto prudencial en Llano.

El saber práctico se caracteriza entonces por la actuación de la voluntad, que decide realizar a partir de la razón práctica, y no teórica. El resultado de lo captado por la razón teórica es pura especulación, es conocimiento de la realidad, y por lo mismo, es abstracción pasiva frente a la realidad. Recuperando la tradición aristotélico-tomista, el entendimiento se define como la facultad humana por la cual captamos la realidad en cuanto tal y juzgamos sobre

11 Llano, C. (1979), p. 71-72. Llano, C. (1979), p. 95.

12 Llano, C. (1979), p. 21. 
ella ${ }^{13}$. Se distingue la razón teórica de la práctica, en lo ya dicho. Aunque el objeto de ambas es la verdad, y las dos aprehenden de la realidad los objetos, en la primera -la teórica- el entendimiento es meramente especulativo y no práctico, está encerrado en la esfera de lo mental, lo pensado, y es incapaz de transformar la realidad porque ese no es su fin ${ }^{14}$. En el caso de la razón práctica, se contempla de modo particular el objeto, y se añade, a lo contemplado por la razón teórica, la noción de bien ${ }^{15}$. Se podría comprender a la razón teórica como el quehacer mental confinado al campo de lo intangible y la abstracción, y la razón práctica como la captación del bien en el objeto particular, que al ser presentado a la voluntad se vuelve apetecible. En palabras de Tomás de Aquino, la razón práctica es segunda respecto de la teórica, y esto es necesario porque la primera da orden a la segunda en cuanto a la aprehensión del bien. La razón teórica distingue los bienes verdaderos, de los aparentes. Para Llano, el entendimiento es sólo especulativo, pero "cuando versa sobre una oportunidad de acción, se encuentra en potencia para ser práctico, es decir, puede servir a la acción práctica. En rigor, diríamos que es un conocimiento especulativo con una relación trascendental -ontológica- a la práctica" ${ }^{16}$. La razón práctica se entiende como ese mismo entendimiento especulativo en el que incide la voluntad, pues "el ser de la acción depende de la voluntad como de su causa eficiente; pero la determinación específica de la acción proviene del entendimiento que dirige la voluntad al modo de su causa formal" ${ }^{17}$.

Cada uno de estos modos del entendimiento da lugar a los dos tipos de conocimiento: el teórico y el práctico, respectivamente. El primero se refiere a lo que se conoce como 'necesario' y que no puede ser de otra manera ${ }^{18}$. Esto quiere decir que el conocimiento de la ciencia se mueve en el ámbito de aquello que no cambia, no es aleatorio, que no está sujeto al devenir, a la irregularidad, ni a las particularidades del contexto, pues los postulados de la razón teórica se ubican más allá de los cambios del mundo fáctico y material. Como diría Aristóteles, "el razonamiento teorético no es práctico ni productivo ni tampoco es buena o mala su verdad o su falsedad (pues esto es, del todo, propio de la actividad intelectual)" 1 . En este sentido, al ámbito científico "co-

13 Llano, C. (1979), p. 96.

14 Llano, C. (1979), p. 97.

15 Aquino, Q.D. De Veritate, q. 3, a. 3, ad. 5.

16 Llano, C. (1979), p. 97.

17 Llano, C. (1979), p. 99.

18 Llano, C. (2005).

19 Aristóteles, EN 1139a. 
rresponden las verdades generales y permanentes"20. El entendimiento práctico -a diferencia del teórico- se refiere a lo que varía, a aquello que puede ser de otro modo, realidades contingentes que pueden ser aleatorias o, al menos, variables ${ }^{21}$. El razonamiento práctico se ocupa de todo el espectro de la realidad cambiante, dentro del cual se engloban buena parte de las acciones y experiencias humanas, y más específicamente aquellas que tienen que ver con las relaciones interpersonales.

La acción directiva es un estudio de la razón práctica, dado que su labor se realiza en el ámbito de lo cambiante, se lleva a cabo dentro de variables en perpetuo movimiento. No puede dirigirse una empresa por medio de leyes o axiomas fijos, ya que éstos no aplican a la particularidad de cada situación, ni responden a las preguntas que presentan las diferentes circunstancias de la realidad, sino que se reducen a describirla o explicarla en sus fenómenos estables. Esto da lugar a la virtud de la prudencia, entendida como la capacidad para saber qué hacer en cada situación particular: "en el aquí y ahora". El director de empresas carece de leyes científicas que orienten su conocimiento, ya que es práctico, su guía rectora es la prudencia. En definitiva, el liderazgo empresarial es más parecido a la improvisación, hay una guía que sirve y se construye de la visión general, hay valores compartidos y la estrategia a seguir, pero el quehacer propio del director de empresas debe generarse conforme se desarrollan los conocimientos ${ }^{22}$.

\section{LOS DESAFÍOS QUE PLANTEA LA IA: UNA APROXIMACIÓN CUALITATIVA}

Existen numerosos mitos en torno a la llamada IA. Para abordar este apartado, hemos realizado un estudio empírico a través de entrevistas en profundidad a seis expertos en el campo de la empresa y la tecnología, tanto de posiciones directivas como técnicas, con el fin de explorar los significados y experiencias en torno al objeto de estudio. Analizamos los temas y subtemas que surgen de las entrevistas desde una perspectiva fenomenológica, que pone énfasis en la experiencia vivida, dando pauta a principios metodologicos sobre cómo interpretar el mundo desde la interaccion de actores sociales, cómo estudiar los modos en que aparecen las configuraciones y cómo se construyen

20 Llano, C. (2005), p. 22.

21 Llano, C. (2005).

22 Heifetz, R. \& Linskt, M. (2002). 
los significados personales sobre el mundo circundante ${ }^{23}$. La muestra para la entrevista se seleccionó entre directores de tecnología de empresas trasnacionales, fundadores de empresas de tecnología, especialmente aquellos que utilicen la IA en sus modelos de negocios, especialistas en el manejo del Big Data, etc., con el objetivo de hacer un análisis comparativo entre la IA y la inteligencia práctica y entender el futuro de la labor directiva en las empresas. Las entrevistas se realizaron entre el 31 de julio y el 14 de septiembre de 2019 en la ciudad de México, y en total alcanzan casi seis horas de grabación.

En primer lugar, conviene destacar la dificultad de precisar una definición precisa sobre IA.

E1: "no hay definición específica de IA, hay una, un poco tautológica en el sentido de que es cualquier método o sistema de cómputo que pueda arremedar o hacer algo atribuible a una inteligencia humana".

E2: "no me he encontrado con una definición única para definir IA, aún hay muchas y no hay consenso sobre cuál es la correcta. Porque hay elementos de la IA que inciden en diferentes áreas y proceso de productivos o no productivos en investigación, etc. Para mí, como ahorita lo veo, lo que trata o hace la IA es programar una estructura lógica de una inteligencia humana, pero de manera colectiva (...) y que además puede programarse con reglas para que aprenda ese proceso, programa o algoritmo".

E5: "la definición ha ido cambiando, porque a medida que la gente tiene mayores expectativas de lo que pueda ser la tecnología la gente se sorprende menos y lo considera menos inteligente artificialmente, yo creo que ese es el primer punto. ¿Qué es? No es una cosa, son muchas, eso es importante, porque hay múltiples distintos tipos de algoritmos para resolver distintos tipos de problemas".

E6: "Es un conjunto de herramientas y metodologías que utilizan los avances tecnológicos para tratar de emular ciertos aspectos del pensamiento intuitivo-predictivo aterrizados a algún proceso de negocio, es decir, debe tener un enfoque productivo, es decir, debe estar orientado a generar rentabilidad".

El diccionario Merriam-Webster define a la inteligencia artificial como "una rama de la ciencia de la computación que intenta simular el comporta-

23 Rodríguez, G., Gil, J., \& García, E. (1999); Galeano, M. (2004). 
miento inteligente" y "la capacidad de una máquina de imitar el comportamiento humano" ${ }^{24}$. Ambos usos han sido estipulados desde el inicio de la ciencia computacional. Por un lado, Alan M. Turing se preguntó en 1950 si las máquinas podían pensar, para responderse reformuló la cuestión de un modo más creativo y postuló sus reglas a través de una hipótesis que se conoce como 'The imitation game ${ }^{25}$. El juego consistía en retar a una persona (interrogador) a descubrir el sexo (masculino o femenino) de dos candidatos que se encontraban ocultos en dos cuartos distintos. Para hacerlo, el individuo debía hacer preguntas que le permitieran ir descifrando si se trataba de un hombre o una mujer quien las respondía. El reto tecnológico, que a Turing le interesaba, consistía en intercambiar a uno de los interlocutores por una máquina y probar cuanto tiempo pasaría antes de que el interrogador, que intentaba adivinar el sexo de las "personas ocultas", se diera cuenta de que en realidad hablaba con un ordenador. Por otro lado, en 1956, John McCarthy, bajo el auspicio del Summer Research Project, en la Universidad de Dartmouth, propuso el término de 'Inteligencia Artificial', que desde entonces es utilizado para definir todo aquel 'proceso realizado por la inteligencia humana' que pueda ser simulado por una máquina ${ }^{26}$. Cada vez que se lograra imitar un proceso humano se podría estipular que las máquinas poseían una 'capacidad intelectual ${ }^{27}$. Hoy, estos dos futuros descritos han sido alcanzados, los 'chatbots' son capaces, por ejemplo, de tomar pedidos de pizzas y resolver dudas de diferentes cuestiones a clientes, sin que estos se den cuenta y son múltiples los usos de robots que sustituyen las labores humanas en actividades técnicas u operativas, como sucede en los almacenes de $\operatorname{Amazon}^{28}$, o los más recientes artefactos que distribuyen los productos en los anaqueles de Walmart ${ }^{29}$. Sin embargo, la pregunta inicial sigue estando vigente: ¿las máquinas pueden pensar?

E1: "muchas de las definiciones nos remiten a la prueba de Turing, en el sentido de que tú puedas atribuir a una máquina un sistema o un comportamiento que sea atribuible a un ser humano sin notar la diferencia específica,

24 Recuperado de: https://www.merriam-webster.com/dictionary/artificial\%20intelligence.

25 Turing, A. M. (1950).

26 Moor, J. (2006).

27 Moor, J. (2006), p. 87. "La visión fue asentada de manera inteligente en la propuesta de la conferencia de 1956: "El estudio se realiza bajo la conjetura de que todos los aspectos del aprendizaje o cualquier otro atributo de la inteligencia puede en principios describirse tan precisamente que una máquina puede construirse para simularlo".

28 Amazon Kiva Robotics, Li, J. \& Liu, H. (2016).

29 Morgan, B. (2019). 
una prueba de Turing que de alguna manera no ha pasado. Un chatbot puede pasar rápidamente la prueba de Turing".

E2: "Si al chatbot, el que lo diseñó le pone 'por favor' y 'gracias', y no se engancha contigo, va a ser mucho mejor que tener un tipo que no sabe decir ni 'por favor' ni 'gracias' y que no se engancha, el robot puede ser cortés siempre, y dar mejor trato de que cualquier humano".

E4: "un chatbot en servicio al cliente, cuando ya no puede solucionar te manda con una persona, y se ahorran el contrato de muchas personas, y esa persona que resuelve el último nivel de soporte debe retroalimentar los casos atípicos que no puede resolver el sistema, para que después él se quede sin trabajo, esto sí está cañón".

Hasta el día de hoy la IA descansa principalmente en procesos denominados algoritmos, mismos que son ejecutados por sistemas de 'machine learning ${ }^{30}$. Los algoritmos funcionan utilizando 'datos' y clasificándolos de acuerdo con una instrucción que ha sido desarrollada por un diseñador. Estos resultados pueden generar -dependiendo el mecanismo en el que se utilicendesde una respuesta o solución informativa, como puede ser alguna recomendación médica o de rutas para un destino, hasta recomendaciones de acuerdo con información recibida por el interlocutor humano y la información existente en la 'nube', como es el caso de procesadores autónomos como 'Siri' o 'Alexa'. Otros procesos de machine learning se encuentran detrás de los robots autónomos, entre los más famosos los automóviles de diferentes marcas que ya circulan en algunas ciudades de Estados Unidos. Algunos otros son brazos mecánicos o simples máquinas que suelen utilizarse, principalmente, en plantas industriales ${ }^{31}$.

E1: "es un caso famoso [el de Watson aplicado al diagnóstico médico], no solo porque logra una certeza mayor que un oncólogo especializado, sino porque la máquina no sufre de ciertos sesgos que el ser humanos sí (...) [en efecto] una de las grandes críticas es que si quien entrena a la máquina es un ser humano, hay un peligro muy grande de que el sistema tenga el sesgo de la persona que lo programó".

E4: “yo veo [el machine learning] como la base de la IA, ya que no es más que emular cómo aprendemos los humanos, diseñar una computadora por prueba y error y por diferentes casos. IA es realmente que una maquina pueda

30 Burrel, J. (2016).

31 El-Namaki, M. S. S. (2018). 
tomar decisiones; ahí el tema es que lo que conflictúa a muchas personas, incluyéndome, es que una programación o computadora es determinística, algorítmica, es decir, tú programas un algoritmo determinístico, y ahí es donde yo me topo con pared acerca de hasta qué punto nombraremos algo inteligente (...) hasta la fecha nosotros no tenemos un algoritmo, o es demasiado, podemos reprogramarlo internamente en el cerebro y a las computadoras les cuesta un poco más este tipo de cuestiones".

E5: "La computadora, teniendo el dato, puede contestar de manera adecuada. Si tú ves a Siri, por ejemplo, como asistente virtual, es el único que tiene un poquito de personalidad, como que te contesta retándote, o intentando poner algo de humor o demás, pero es algo que no tiene ninguna limitación técnica para que otros lo hagan, realmente la interacción, si la información está, tú puedes contestar como quieras. Pero eso no significa que haya empatía, sino que cuando sabes que hay alguien que está triste, quizá le intentas hacer otras preguntas, pero a la computadora no le importa".

Existen también robots con apariencia humana. Los más avanzados interlocutores informáticos son quizá los desarrollados por Hanson Robotics, propiedad del diseñador robótico David Hanson, empresa responsable del desarrollo de Bina48, una androide que fue fabricada a partir de Bina Aspen, esposa del diseñador, de quien tomó sus memorias, sueños, emociones y creencias $^{32}$; y 'Sophia', la primera robot androide con 'nacionalidad saudí' que a través del uso del machine learning es capaz de conversar con seres humanos de manera natural, emular gestos que denotan estados emocionales e incluso llegar a tener 'agrado' por personas o deportes, es admiradora de Cristiano Ronaldo y Mohamed Salah ${ }^{33}$. Más allá de la curiosidad que despiertan, nos permiten entender los múltiples usos que se puede hacer de la llamada IA, cada una de ellas refleja posibilidades de un futuro en el que las máquinas y los seres humanos deben aprender a convivir de manera habitual. Más allá del grado de avance tecnológico que denotan estos inventos y que seguramente derrotarían a muchos 'interrogadores' en el juego propuesto por Turing, la pregunta sigue abierta.

E2: "se está tratando de diseñar estas inteligencias a veces con forma y trato humanos, pero yo creo que un tema muy importante es cómo exponencialmente está evolucionando (...) Ahora se está buscando, por ejemplo, en estos robots que interactúan en la casa, por ejemplo, Alexa, se le está tratando

32 Robotics, H. (2019). 
de dotar para que sean más humanos (...) y se le tiene que dotar con capacidad de interacción más humana, entender mucho el contexto y eso los humaniza, es decir, sí hay una tendencia clara a humanizar la IA porque va interactuar con nosotros (...) Y cuando menos nos demos cuenta será lo más común tener una plática con una Alexa o Siri y no vas a sentir que estuviste platicando con una máquina porque realmente te contestará bien y te sigue la conversación”

E1: "no hemos sido capaces de generar inteligencia multipropósitos (...) creo que de eso estamos aún muy lejos (...) a las máquinas les cuesta trabajo percibir como tú percibes: en sentido olfato, oído, vista, y aproximar eso de una manera muy rápida, si se lograra inseminar todo eso con un alto poder de procesamiento se puede lograr ese asunto (...) una máquina se puede hacer inteligente sobre una función, pero no 'multi-inteligente' al grado del ser humano".

E4: "definamos qué es inteligencia para ti y para mí; para mí es que puedas razonar y tener conciencia e intuición y muchas cuestiones que tomas en una decisión, pero para mí, por ahora, una computadora no es inteligente, es una decisión bien informada".

E5: "El ser humano puede tener varias preocupaciones al mismo tiempo, es multitask, y eso es lo que aún no se ha podido llevar a cabo en IA, son procesos decisivos de impacto que ésta no puede hacer, están limitadas a una decisión del ser humano".

En efecto, la mayoría de los expertos en las ciencias computacionales niegan la posibilidad de que las máquinas sean inteligentes, al menos al modo de la inteligencia humana. Distinguen entre dos tipos de IA: la fuerte y al débil. La primera se refiere a lograr un programa de cómputo capaz de funcionar independientemente del diseñador; del mismo modo que la capacidad humana, sería equivalente a tener una conciencia y ser capaces de un pensamiento abstracto general, capaces de realizar distintos tipos de tareas con el mismo 'procesador'. Por su parte, la IA débil depende de la programación humana para ejecutar sus funciones ${ }^{34}$. Las de segundo tipo suelen ser programas especializados en alguna función, como sucede con el programa de computo desarrollado por Google denominado $A l p h a G o^{35}$, que en marzo del 2016 derrotó al mejor jugador del mundo de Go, un juego que a pesar de contar con reglas

\footnotetext{
33 Robotics, H. (2019).

34 Searle, J. (1980).

35 Scott, R., Beck, A. \& Papke Jr., D. (2017).
} 
sencillas tiene más combinaciones que átomos en el universo, y es mucho más complejo que el ajedrez ${ }^{36}$. Este grado de especialidad es difícil de alcanzar por el cerebro humano, hay pocas personas en el mundo que tienen la capacidad de competir con un ordenador de esta naturaleza. Pero como se ha explicado antes, es un tipo de IA débil, ya que el mismo programa sería derrotado por un niño si se le pusiera a competir en damas chinas. La IA 'débil' logra, hasta cierto punto, pasar la prueba de Turing, pero en realidad es insuficiente, porque generalmente las personas son capaces de distinguir si están jugando con una persona o con una máquina, hasta ahora, a pesar que desde 1990 hay un premio de hasta 100.000 dólares por un programa que logre superar la prueba de Turing, no ha sido posible desarrollar un programa que logre engañar a un ser humano ${ }^{37}$, mucho menos que las máquinas lleguen a tener conciencia o les sea posible utilizar la misma potencia (en cuanto capacidad tecnológica) para diferentes objetos o problemas a resolver.

E1: "aunque débil en su inteligencia, Waze termina siendo buenísimo y mejor que tú para elegir rutas, porque ha tenido la experiencia de todas las personas que toman diferentes rutas, y entra más personas usen Waze, mejor aprende, entonces las máquinas tienen uso de poder prudencial”.

E2: "hay que tener cuidado, porque si decimos 'aprender', nos imaginamos un proceso creativo y autónomo, como lo tendría una capacidad del ser humano, que es la única especie que lo tiene; la realidad es que aprende a través de un programa de aprendizaje que un humano le escribió".

E2: "al final si vemos elementos comunes de todas estas comunicaciones independientemente de la industria en la que están, al centro hay un programa diseñado y codificado por humanos, donde se ponen reglas para operar ciertas instrucciones, y el otro elemento que tienen estas en común o las más avanzadas que tienen la capacidad de aprender, y no es que aprendan, no es que haya un código de aprendizaje estándar que aprenda como aprendería el humano, sino que ahí hay que diseñar y codificar cómo aprender; 'si pasa esto se hace esto', y se ponen las reglas y así es como también pudiera parecer que opera como lo haría una persona".

Las máquinas pueden ejecutar programas con una gran capacidad, profundidad y exactitud, siempre y cuando éstos ocurran sobre una misma línea de trabajo, por ejemplo, resolver un crucigrama, responder preguntas cuya búsqueda dependa de ciertos datos que antes le han sido configurados o cuyo

36 Scott, R., Beck, A. \& Papke Jr., D. (2017). 
algoritmo sea capaz de agregar o aprender, pero le resulta imposible tener un objetivo propio o discernir sobre distintos tipos de problemas. Incluso los programas desarrollados por Hanson Robotics son en realidad procesadores de información muy potentes pero que no se alejan mucho del funcionamiento de 'chatbot' o un procesador de lenguaje con más capacidad de almacenamiento, acceso a la información y velocidad en el procesamiento de datos, pero que una vez que se entiende cómo funcionan estos programas se 'pierde la magia ${ }^{38}$. La pregunta de si las máquinas pueden pensar se ha cambiado a una más sencilla de responder: ¿cómo hacer que las máquinas piensen en este determinado asunto? No es que se asuma que lo hacen, sino que esta reformulación permite a los desarrolladores concentrarse en un solo aspecto de la realidad y resolverlo en profundidad, sin preocuparse por su capacidad para imitar la inteligencia humana en su totalidad. Los procesos deliberativos en la máquina no nacen de un interés o motivación de la computadora, sino que son establecidos con reglas fijas por parte de un programador ${ }^{39}$, estos algoritmos obtienen la información de dos fuentes: (1) de la información que existe en 'la nube', que suelen ser procesadores de información integrados en una computadora, o (2) de sensores autónomos que tienen contacto con el medio ambiente, conocidos como robots autónomos.

E2: "ahí es donde se da el aprendizaje, para los robots, yo creo que la base es primero programar lo que puede hacer y el loop de feedback en función de lo que regresa en una vez o dos y demás máquinas, aplica la estadística para tomar una decisión de si esto lo convierto”.

E4: "no hay computadoras que se puedan reprogramar, siempre necesitan de la mano humana (...) el cerebro humano ha tenido si no billones por lo menos cuatro millones o quién sabe, el ser humano ha tenido mucho tiempo para desarrollarse o formular esa operaciones complejas, la computadora tiene 60, 30 ó 40 años, no sé si en 100 años la cosa cambie. Es decir, la computadora es determinística, no tiene conciencia”.

E5: "hoy en día partes del dato, porque en la IA automática, donde tú le pasas la información y te genera el algoritmo, no hay un criterio humano y no sabes qué base se está tomando, pero luego lo pruebas y dices: 'mira, sí funciona"”.

37 Armstrong, A. (2015).

38 Armstrong, A. (2015).

39 Burrel, J. (2016). 
E6: "para que haya una idea de que habrá IA para ' $x$ ' o ' $y$ ' cosa, se te tiene que ocurrir la idea, y eso solo lo hace una persona, por ejemplo, en la industria donde yo trabajo encuentras la oportunidad de sustituir personas por robots, que es lo típico de IA, y todo parte de una idea, y esto viene de un caso de negocio, y esta idea se estructura de forma adecuada, y debe integrar procesos, tecnología, herramientas y gente".

Aunque ciertamente hay robots autónomos que interactúan con diferentes ambientes y por lo tanto deben ser capaces de 'deliberar' para alcanzar su objetivo, estos actos deliberados deben estar motivados por un agente externo que es quien programa con vistas a conseguir un objetivo; en ninguna de las consideraciones actuales se entiende que la autonomía implica capacidad de una motivación intrínseca por parte de los robots. El estado actual de estas tecnologías implica el trabajo conjunto entre IA ('machine learning') y robótica para lograr una auténtica acción deliberada ${ }^{40}$, sin embargo hasta el día de hoy es imposible lograr una visión amplia -ni por parte de los investigadores, ni por parte de la robótica 'IA Fuerte'- que permita un progreso coherente o integrado en los robots autónomos. Estos autores ${ }^{41}$ señalan seis funciones deliberativas que pueden realizar los mecanismos autónomos: (1) Planeación, que consiste en combinar la predicción y la búsqueda de información con base a establecer una ruta de dirección para lograr algún propósito; (2) Actuación, crear a partir de lo planeado una serie de comandos para ejecutar; (3) Observación, detecta y reconoce diferentes aspectos de la realidad y los considera para completar la tarea. Estos elementos aparecieron después de lo planeado; (4) Monitoreo, compara las discrepancias entre lo planeado y la realidad, y realiza ajustes; (5) Seguimiento de los objetivos, que significa que va actualizando el nivel de logro del objetivo o misión; y, (6) Aprendizaje, le permite adquirir, adecuar, adaptar y mejorar en el futuro a través de la experiencia. Estas funciones pueden compararse con la labor directiva en la empresa, tomando en cuenta la consideración de que hasta hoy sólo hay robots autónomos que realizan estas funciones con vistas a un objetivo concreto, como los automóviles autónomos, que no serían capaces de utilizar los mismos criterios con vistas a otros objetivos. Como consecuencia, se pueden vislumbrar importantes consecuencias para el futuro del trabajo, especialmente de cara a su configuración social.

40 Ingrand, F. \& Ghallab, M. (2014).

41 Ingrand, F. \& Ghallab, M. (2014). 
E1: "por mucho tiempo creí que la tecnología iba a solucionar todos los problemas humanos, pero esta creencia ya está en duda, porque este asunto te reta... por ejemplo, la velocidad en la que pueden desaparecer empleos puede ser más rápida que la capacidad de generar nuevos empleos (...) me parece que ahora la tecnología puede destruir más rápido las cosas de lo que puede crear (...) considero que en un futuro efectivamente el ser humano estará muy provocado a cambiar muy fuertemente lo que ha hecho toda su vida, yo sí creo, como Bill Gates, que la solución es una renta pareja para todos, y que cada quien se dedique a hacer lo que mejor le convenga: escribir música en una hamaca, escribir poesía o pintar".

E2: "Yo creo que [la IA] tendrá que ser regulada, porque si no, nos va a rebasar, como sucedió con el internet, que se movió más rápido que la capacidad de regulación, deber ser regulada porque provoca nuevos aspectos, nos cambia éticos y morales. El otro día vacilaba con mi esposa, pues en el futuro si ando con un robot ¿eso se considerará infidelidad o no? Pues a mi esposa no le cayó bien la voz de Alexa. Son planteamientos válidos para discutir lo ético, lo moral, y al final te lleva a que ciertas cosas se tengan que regular porque se transforma nuestro entorno, como ha sucedido en todas las revoluciones industriales. Yo creo que lo que va a suceder es que se va a mover más rápido la tecnología, la capacidad de regular y va a ser esa carrera de cómo empezar a controlarlo".

E4:"Cada vez que hay un cambio tecnológico importante, hay un desplazamiento de personas, es inevitable (...) sí va haber trabajos desplazados por machine learning, seguro, si le pones un sensor de visión a un carro, por ejemplo, por supuesto que habrá desplazamientos de trabajos que serán desplazados por la máquina, pero no quiere decir que sea porque sean inteligentes sino porque no se cansan, no se quejan, no se van a un sindicato, no te cobran, es un tema completamente diferente económico".

E5: "la IA tiene que ser regulada, Si tú no le pones reglas de juego a esto, mostrándole lo que puedes o no hacer, como es el caso de hoy en día de la selección del personal (...) pues puede ser que el único criterio válido es si tiene la capacidad técnica para desempeñar el puesto, pues si ese es el criterio, todavía se puede utilizar la IA para determinar si la experiencia es verdaderamente relevante, si se parece al puesto que queremos hacer, si los años de experiencia son suficientes, si el puesto del título anterior es parecido al que queremos, etc., pero sin reglas esto no funciona". 
E6: "[hay un enfoque que] tiene que ver con bienestar social y todos los avances que se hagan en cuestión de IA cada vez más aplicados, por ejemplo, en aspectos de salud, eso es responsabilidad social, lo deberíamos aplicar a cosas así y ahí no deberíamos tener ningún límite (...) pero a veces solo ve el bien propio de ciertos países o personas, por ejemplo, al nivel militar, de armas, etc., ahí si se debe regular".

\section{CONCLUSIONES}

Este trabajo representa una primera aproximación a un tema de gran interés, pero que, debido a la rapidez con que se han sucedido los cambios tecnológicos en los últimos años, supera aún nuestra capacidad para comprender las consecuencias que traerá para el futuro del trabajo. En parte promovidas por una visión mítica proveniente de la ciencia ficción más que del avance real del fenómeno, rodean al tema preocupaciones antropológicas muchas veces apocalípticas. El análisis de las percepciones de profesionales expertos en la materia que abordó empíricamente este trabajo ayuda a disipar tales tensiones, sin por eso minimizar el desafío que esta situación histórica representa.

De acuerdo al marco teórico elegido, y de cara a entender el futuro de la acción directiva en las empresas, uno de los aspectos más importantes que se deben destacar es el papel de la voluntad en la toma de decisiones. Todos los entrevistados han destacado que la máquina es incapaz de innovar y de tener intención, y esto se debe a que no se ha considerado en la programación de los algoritmos el papel de la voluntad en la acción. Ante esta diferencia es preciso resaltar el juego que se da entre voluntad y entendimiento con vistas a decidir y llevar a cabo una tarea de cualquier tipo. Si bien ha quedado ya estudiada la diferencia entre las dos potencias de la parte racional: la teórica y la práctica es pertinente estudiar el papel de la voluntad. Primero, debe quedar claro que "la voluntad sigue al entendimiento, no le precede, y apetece necesariamente aquello que se le presenta como un bien que sacia por completo el apetito; pero elige libremente entre aquellos otros bienes cuya apetencia es propuesta por un juicio variable. Por consiguiente, la elección sigue al último juicio práctico, pero de la voluntad depende que dicho juicio sea o no el último" ${ }^{2}$. La incidencia de la voluntad es en el juicio último del entendimiento. La inteligencia práctica se concreta gracias a la relación entre entendimiento

42 García López, J. (2006). 
y voluntad, y esto en dos momentos distintos: uno pasivo, que es cuando la voluntad le presenta la oportunidad de acción, para que ésta lleve a cabo una decisión después de un proceso deliberativo; y un segundo momento, activo, cuando 'impera' a la misma voluntad para que ejecute la decisión tomada de acuerdo a la estrategia establecida ${ }^{43}$.

Después de que el entendimiento juzga, especulativamente, sobre la oportunidad, y delibera para la fijación de un objetivo, toda persona está en potencia inmediata a la acción. Es gracias a este juicio que la voluntad decide llevar a cabo la tarea. Una vez hecha la decisión, y "mientras ésta subsiste dinámicamente", el entendimiento "dirige" la ejecución de la acción. "Lo que significa que el entendimiento es práctico no (sólo) en cuanto receptor de oportunidades (practicidad pasiva), sino en cuanto director de la ejecución de la acción ya decidida por la voluntad" ${ }^{\prime 4}$. El ser de la acción depende de la voluntad como de su causa eficiente, y del entendimiento como causa formal. El acto del entendimiento práctico, absolutamente considerado, es el "imperio" en la voluntad, y deriva del hábito intelectual de la prudencia. Antes de proseguir, es importante tener clara la distinción entre hábitos intelectuales y virtudes de la voluntad.

Es importante resaltar que la razón teórica es la facultad básica ${ }^{45}$, es decir, sin ésta no hay posibilidad de decisión, la razón práctica es una ampliación de la primera, por esto la IA contribuye a la toma de decisiones. Esto denota la unidad de la facultad, pero también el orden. La razón práctica sigue en orden a la teórica dado que primero debe contemplar al ser, o a la realidad. En este caso se podría hablar de las oportunidades del mercado, y no podría decidir e imperar si no fuera porque la especulativa conoce antes. Por esto se dice que un apetito es recto cuando sigue la recta razón ${ }^{46}$. Aunque es verdad que las dos tienen como fin la verdad, cada una la conoce de modo distinto. La intelectiva en cuanto necesaria, mientras que la deliberativa mirando a la acción.

Una segunda conclusión relevante a partir de lo estudiado es el deterioro de la capacidad de aprendizaje en el director, lo que se considera la formación del criterio de decisión a través del hábito intelectual. Se está generando en

\footnotetext{
43 Gilson, E. (1964).

44 Llano, C. (1979), p. 100.

45 Pieper, J. (1974).

46 De Aquino, T. (2010).
} 
estos tiempos una especie de "fe tecnológica", que puede mermar el desarrollo intelectual del usuario. De tal modo que se pierde el hábito de pensar. En la palabra 'bábito' cabe un posible equívoco terminológico que debe ser también esclarecido de entrada. No se debe entender lo que usualmente se conoce por habilidad, costumbre, conducta, práctica, uso, etc. La palabra está tomada, más bien, en su significado clásico, es decir, según su acepción usual tanto en la filosofía griega como en la medieval, a saber, como "una perfección adquirida e inmanente en la inteligencia que realza la perfección de esta facultad y la capacita para conocer más de lo que antes conocía" ${ }^{47}$. Siguiendo a los clásicos, Llano establece la acción directiva en la razón práctica, que, por intervención de la voluntad, mira al objeto en lo que tiene de bueno, de apetecible ${ }^{48}$, y, de los dos hábitos posibles de ésta, la asigna a la prudencia.

Aunque la razón teórica precede a la práctica, la segunda es capaz de dirigir la acción ${ }^{49}$. Esta capacidad se denomina imperio, prudencia, en la razón práctica $^{50}$. A este hábito Llano lo denomina el propio de la acción directiva. No indica que sólo sea para esto, sino que la dirección de empresas sólo puede llevarse a cabo desde este hábito intelectual. La IA hoy está muy lejos de lo que se puede considerar un acto prudencial. De momento se resalta su capacidad de reunir información y evaluarla de acuerdo con unos criterios que han sido establecidos por el agente, mismos que pueden tener omisiones de origen porque no consideran todas las circunstancias del momento actual, sino que son elementos predictivos, pero no definitivos.

La distinción hecha entre razón teórica y razón práctica es importante para evitar que la acción sea o bien pura especulación, o una ejecución sin pensamiento, como se pretende realizar por la IA. La facultad intelectiva realiza los dos conocimientos, por lo que se concluye que la acción directiva es posible antropológicamente, mientras que no lo es por una máquina que sólo es captar la realidad de manera general. La razón teórica requiere de la voluntad para volverse práctica; ver lo bueno, en el objeto, sólo es posible por la intervención de la facultad volitiva, sin ella nada de lo que sucede en la empresa sería posible, no habría resultados concretos, sino conocimientos abstractos, posibilidades algorítmicas. El director de empresa requiere de la voluntad para mirar lo que le interesa de la realidad, lo concreto y práctico. Gracias a que

\footnotetext{
47 Sellés, J. F. (1998).

48 Llano, C. (1979), pp. 99-101.

49 EN 1138b-1139 a.

50 Llano, C. (1979).
} 
esto se da a nivel de facultades, y no como mera tipología -estilo, formas de ser- es posible plantear una formación de la razón práctica y de la voluntad. En definitiva, la dirección es un hábito de la razón práctica -la prudencia, en concreto- que puede perfeccionarse a través de la experiencia, algo que las máquinas no podrán más que emular, y siempre de manera instrumental.

\section{BIBLIOGRAFÍA}

Aristóteles (2015), Etica a Nicómaco. (J. L. Calvo Martínez, Trad.), Alianza, Madrid.

Armstrong, Alex (4 de mayo de 2015), i-programmer. Recuperado el 15 de septiembre de 2019, de https://www.i-programmer.info/babbages-bag/297artificial-intelligence.html

Bachmann, Claudius; Habisch, André y Dierksmeier, Claus (2017), "Practical Wisdom: Management's No Longer Forgotten Virtue", fournal of Business Ethics, vol. 153, n⿳ 1, pp. 147-165.

Bauman, Zygmunt (2004), Work, Consumerism and the New Poor (Issues in Society), McGraw-Hill Education, Londres. bridge.

Beck, Ulrich (2000), The Brave New World of Work.,Polity Press, Cam-

Brown, Philipp y Keep, Ewart (2018), "Rethinking the Race Between Education \& Technology", Issues in Science and Technology, vol. 35, $\mathrm{n}^{\mathrm{o}}$ 1, pp. 3139.

Burrel, Jenna (2016), "How the Machine 'Thinks': Understanding Opacity in Machine Learning Algorithms", Big Data \& Society, pp. 1-12.

Chamberlain, James A. (2018), Undoing Work, Retbinking Community. A Critique of the Social Function of Work, ILR Press, Ithaca and Londres.

De Aquino, Tomás (1999), De Veritate (Cuadernos de Anuario Filosófico, Serie Universitaria $n^{\circ}$ 78). (J. F. Sellés, Trad.) Servicio de Publicaciones de la Universidad de Navarra, Pamplona.

De Aquino, Tomás (2006), Summa Theologiae, Cambridge University Press, Cambridge.

De Aquino, Tomás (210), Comentario a la ética a Nicómaco de Aristóteles, (A. Maella, Trad.), Euns, Pamplona. 
Elliott, Stuart W. (2018), "Artificial Intelligence, Robots, and Work: Is This Time Different?", Issues in Science and Technology, vol. 35, $\mathrm{n}^{\circ}$ 1, pp. 40-44.

El-Namaki, M. Sameh (2018), "How Companies are Applying AI to Business Strategy Formulation”, Scoledge International Fournal of Business Policy \& Governance, vol. 5, nº 8, pp. 77-82.

Galeano, María Eumelia (2004), Diseño de proyectos de investigación cualitativa, Fondo Editorial Universidad EAFIT, Bogotá.

García López, Jesús (2006), "Entendimiento y voluntad en el acto de la elección", Anuario Filosófico, vol. 10, nº 2, pp. 93-114.

Gilson, Etienne (1964), Santo Tomás de Aquino, Aguilar, Madrid.

Granger Scott R.; Beck, Andrew H. y Papke Jr., David J. (2017), “AlphaGo, Deep Learning, and the Future of the Human Microscopist", Archives of Pathology \& Laboratory Medicine, vol. 141, $\mathrm{n}^{\circ}$ 5, pp. 619-621.

Hanson Robotics (2019), “About Bina”, https://www.hansonrobotics.com/bina48-9/ (Recuperado el 24 de septiembre de 2019).

Heifetz, Ronald y Linskt, Marty (2002), "A Survival Guide for Leaders", Harvard Business Review, vol. 80, n 6, pp. 65-74.

Ingrand, Felix y Ghallab, Malik (2014), "Deliberation for Autonomous Robots: A Survey", Artificial Intelligence, vol. 247, pp. 10-44.

Li, Jun-tao y Liu, Hong-jian (2016), "Design Optimization of Amazon Robotics", Automation, Control and Intelligent Systems, vol. 4, n 2, pp. 48-52.

Llano, Carlos (1979), Análisis de la Acción Directiva, Limusa, México.

Llano, Carlos (1990), El trabajo directivo y el trabajo operativo en la empresa, Cuadernos de Empresa y Humanismo, no 9, Servicio de Publicaciones de la Universidad de Navarra, Pamplona.

Llano, Carlos (2005), “Aprendizaje de la ciencia y de la prudencia”, Pensamiento y Cultura, vol. 8, n ${ }^{\circ}$ 1, pp. 13-29.

Moor, James (2006), "The Dartmouth College Artificial Intelligence Conference: The Next Fifty Years", AI Magazine, vol. 27, nº 4, pp. 87-91.

Morgan, Blake (2019), "Slow Your Roll Robots: Not Everyone Is Excited About Walmart's Robots", Forbes, https://www.forbes.com/sites/blakemorgan/2019/09/03/slow-your-roll-robots-not-everyone-is-excited-about-walmarts-robots/\#6d1430233b3b, (Recuperado el 23 de septiembre de 2019). 
Pieper, Josef (1974), EL descubrimiento de la realidad, RIALP, Madrid.

Rodríguez, Gregorio; Gil, Javier y García, Eduardo (1999), Metodología de la investigación cualitativa, Aljibe, Málaga.

Scalzo, Germán (2018), "Virtue Ethics and the Common Good in Production and Manufacturing", en Sison, Alejo José; Ferrero, Ignacio y Guitián, Gregorio, Virtuous Business for the Common Good, Routledge, Londres, pp. 8298 .

Scott R., Granter; Beck, Andrew H. y Papke Jr, David J. (2017), "AlphaGo, Deep Learning, and the Future of the Human Microscopist", Archives of Pathology \& Laboratory Medicine, vol. 141, n 5, pp. 619-621.

Searle, John R. (1980), "Minds, Brains, and Programs", Behavioral and Brain Sciences, vol. 3, n ${ }^{\circ}$ 3, pp. 417-457.

Sellés, Juan Fernando (1998), Hábitos y Virtudes, Servicio de Publicaciones de la Universidad de Navarra, Pamplona.

Sison, Alejo José y Hühn, Martin P. (2018), "Practical Wisdom In Corporate Governance”, en Sison, Alejo José; Ferrero, Ignacio y Guitián, Gregorio, Busines Ethics: A Virtue Ethics and Common Good Approach, Routledge, Nueva York.

Strauss, Anselm y Corbin, Juliet M. (1998), Basics of Qualitative Research. Techniques and Procedures for Developing Grouned Theory, Sage Publications, Thousand Oaks.

Turing, Alan M. (1950), "Computing Machinery and Intelligence", Mind, vol. 49 , pp.433-460.

Vallor, Shanonn (2016), Technology and the Virtues: a Philosophical Guide to a Future Woth Wanting, Oxford University Press, Nueva York. 